\title{
Convective Cores in Stellar Models
}

\author{
I.W. Roxburgh and M. Monteiro \\ Astronomy Unit, Queen Mary and Westfield College, University of London
}

\begin{abstract}
The condition for homogeneous radiative stellar models to be marginally stable to convection at the centre is investigated for the family of models where the opacity $K$ and energy generation $\varepsilon$ are given by power laws in temperature and density $k=\kappa_{0} \rho^{\alpha} T-\beta, \varepsilon=\varepsilon_{0} \rho T \eta$. The Naur-Osterbrock (1953) condition $6 \eta>6+10 \beta-15 \alpha$ is a necessary but not sufficient condition. A better estimate is obtained by taking the effective polytropic index $n=d \log P /$ dlogT -1 to be a linear function of temperature $T$ throughout the model. This gives the condition
\end{abstract}

$$
6 \eta=10 \beta-15 \alpha+\frac{12+4 \beta}{1+\alpha}
$$

The predictions of this condition agree well with results for a set of stellar models $0 \leq \alpha \leq 1,0 \leq \beta \leq 5$.

\section{Analysis.}

The equations of stellar structure for homogeneous radiative stellar models with an ideal gas equation of state and the opacity and energy generation given by power laws are:

$$
\begin{gathered}
\frac{\mathrm{dP}}{\mathrm{dr}}=-\frac{\mathrm{GM}_{\mathrm{r}}}{\mathrm{r}^{2}} \rho \quad \frac{\mathrm{dT}}{\mathrm{dr}}=-\frac{3 \kappa \rho \mathrm{L}_{\mathrm{T}}}{16 \pi a c \mathrm{~T}^{3} \mathrm{r}^{2}} \quad \frac{\mathrm{dM}_{\mathrm{r}}}{\mathrm{dr}}=4 \pi \mathrm{r}^{2} \rho \quad \frac{\mathrm{dL}}{\mathrm{dr}}=4 \pi \mathrm{r}^{2} \varepsilon \rho \\
\mathrm{P}=\frac{\Re}{\mu} \rho \mathrm{T} \quad \kappa=\kappa_{0} \rho^{\alpha_{T}} \mathrm{~T}^{-\beta} \quad \varepsilon=\varepsilon_{0} \rho \mathrm{T}^{\eta}
\end{gathered}
$$

Dimensionless variables $\theta, \pi, \phi, \lambda, \zeta$ are defined by $\mathrm{T}=\mathrm{T}_{\mathrm{c}} \theta, \mathrm{P}=\mathrm{P}_{0} \pi, \mathrm{M}_{\mathrm{r}}=\mathrm{M}_{0} \phi$, $L=L_{0} \lambda, r=R_{0} \zeta$ where $T_{c}$ is the central value of $T$, and $P_{0}, M_{0}, L_{0}, R_{0}$ are suitably chosen scaling constants, the equations of stellar structure reduce to:

$$
\frac{\mathrm{d} \pi}{\mathrm{d} \zeta}=-\frac{\pi \phi}{\theta \zeta^{2}} \quad \frac{\mathrm{d} \theta}{\mathrm{d} \zeta}=-\frac{\pi^{1+\alpha} \lambda}{\theta^{4+\alpha+\beta} \zeta^{2}} \quad \frac{\mathrm{d} \phi}{\mathrm{d} \zeta}=\frac{\pi \zeta^{2}}{\theta} \quad \frac{\mathrm{d} \lambda}{\mathrm{d} \zeta}=\pi^{2} \theta^{\eta-2} \zeta^{2}
$$

The central boundary conditions take the form $\theta=1, \pi=\pi_{0}, \phi=0, \lambda=0, \zeta=0$. With $g_{0}=\pi_{0}^{2+\alpha}$ the solution for $\theta$ and $\pi$ can be developed as a series in $\zeta$ as

$$
\begin{gathered}
\theta=1-\pi_{0} \mathrm{~g}_{0} \zeta^{2}+\frac{\pi_{0}^{2} \mathrm{~g}_{0}}{360}\left[11+5 \alpha+\mathrm{g}_{0}(3 \eta-26-5 \alpha-5 \beta)\right] \zeta^{4}+\ldots \\
\pi=\pi_{0}-\frac{\pi_{0}^{2}}{6} \zeta^{2}+\frac{\pi_{0}^{3}\left(1-\mathrm{g}_{0}\right)}{45} \zeta^{4}+\ldots
\end{gathered}
$$

The polytropic index $\mathrm{n}$ is defined by

$$
\mathrm{n}+1=\frac{\mathrm{d} \log \mathrm{P}}{\mathrm{d} \log \mathrm{T}}=\frac{\mathrm{d} \log \pi}{\mathrm{d} \log \theta}=\frac{\theta}{\pi} \frac{\mathrm{d} \pi}{\mathrm{d} \zeta} \frac{1}{\mathrm{~d} \theta / \mathrm{d} \zeta}=\frac{\theta^{4+\alpha+\beta} \phi}{\pi^{1+\alpha} \lambda}
$$


which therefore has the series expansion

$$
\mathrm{n}+1=\frac{1}{\mathrm{~g}_{0}}\left\{1+\frac{\mathrm{p}_{0}}{60}\left[22+10 \alpha+2 \mathrm{~g}_{0}(3 \eta-26-5 \alpha-5 \beta)-6\left(1-\mathrm{g}_{0}\right)\right] \zeta^{2}+\ldots \ldots\right.
$$

If the centre is marginally unstable to convection then $n+1=2.5$ at $\zeta=0, \mathrm{~g}_{0}=2 / 5$ and the series expansion for $(n+1)$ in powers of $(1-\theta)$ and the value of $d(n+1) / d \theta$ are

$$
\begin{aligned}
& \mathrm{n}+1=\frac{5}{2}-\frac{1}{4}(6+10 \beta-15 \alpha-6 \eta)(1-\theta)+O\left((1-\theta)^{2}\right) \\
& \frac{\mathrm{d}(\mathrm{n}+1)}{\mathrm{d} \theta}=\frac{1}{4}(6+10 \beta-15 \alpha-6 \eta) \quad \text { at } \theta=1, \zeta=0 .
\end{aligned}
$$

The Naur-Osterbrock (1954) condition follows from requiring that $\mathrm{d}(\mathrm{n}+1) / \mathrm{d} \theta<0$ at $\zeta=0$

$$
6 \eta>6+10 \beta-15 \alpha
$$

This is only a necessary condition. A more accurate condition is obtained by requiring that $d(n+1) / d \theta$ is such that $(n+1)$ increases from 2.5 at the $\theta=1$ to the surface value at $\theta=0$ (cf Roxburgh 1985). The value of $n+1$ in the surface layers is readily determined since as $\theta \rightarrow 0, \lambda \rightarrow \lambda_{0}, \phi \rightarrow \phi_{0}$ where $\lambda_{0}$ and $\phi_{0}$ are constants, hence

$$
\begin{gathered}
\frac{\mathrm{d} \pi}{\mathrm{d} \theta} \rightarrow \frac{\theta^{3+\alpha+\beta} \phi_{0}}{\pi^{\alpha} \lambda_{0}}, \quad \pi \rightarrow \theta^{(4+\beta+\alpha) /(1+\alpha)} \frac{(1+\alpha) \phi_{0}}{(4+\beta+\alpha) \lambda_{0}}, \quad \text { as } \theta \rightarrow 0 \\
\mathrm{n}+1 \rightarrow \frac{4+\beta+\alpha}{1+\alpha} \quad \text { as } \theta \rightarrow 0
\end{gathered}
$$

If the centre is marginally stable to convection then $(n+1)=2.5$ at $\theta=1$; hence the average value of $d(n+1) / d \theta$ throughout the model is

$$
\frac{d(\overline{n+1})}{d \theta}=\frac{5}{2}-\frac{4+\beta+\alpha}{1+\alpha}
$$

If we now take $d(n+1) / d \theta$ to be constant throughout the model, this mean value is equal to the value at the centre, hence

$$
6 \eta=10 \beta-15 \alpha+\frac{12+4 \beta}{1+\alpha}
$$

This is the new criterion to estimate whether stellar models have convective cores.

\section{Results.}

The equations of stellar structure in the dimensionless form given above were solved to determine $\eta$ such that $n+1=2.5$ at $\zeta=0$, given $\alpha$ and $\beta$. The solutions are very sensitive to the assumed value of $\eta$; if $\eta$ is too small $(n+1)$ rapidly decreases, whereas if $\eta$ is too large $(n+1)$ rapidly increases, the requirement that $n+1$ should tend to 
$(4+\alpha+\beta) /(1+\alpha)$ for large $\zeta$ (small $\theta)$ determines $\eta$ without too much difficulty. The results are shown in Figure 1. In Figure 2 these numerical results are compared with the predictions of the Naur-Osterbrock condition and the new condition. The predictions of the new condition are in satisfactory agreement whereas the Naur-Osterbrock condition underestimates the value of $\eta$ required for the existence of a convective core.

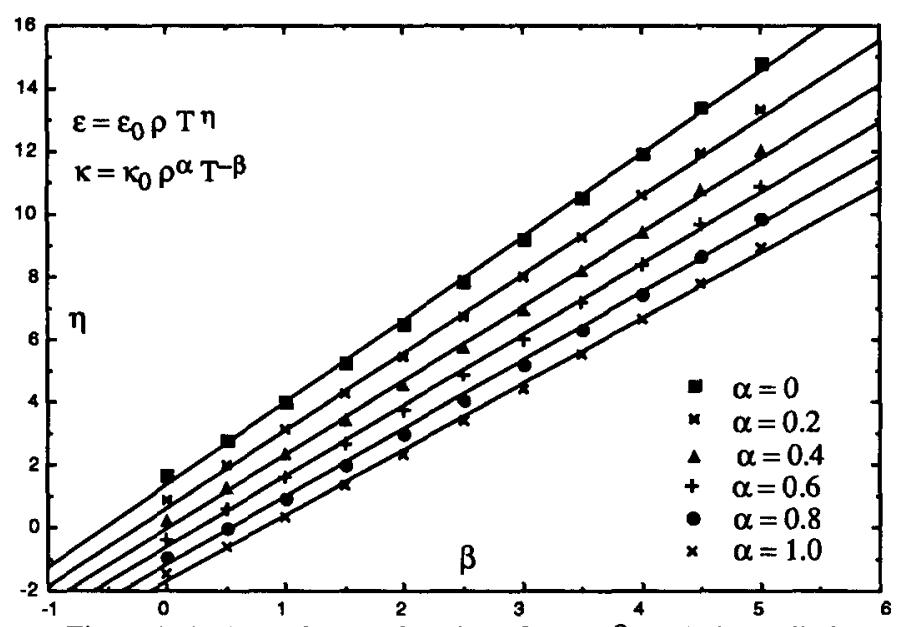

Figure 1. Values of $\eta$ as a function of $\alpha$ and $\beta$, such that radiative stellar models are marginally unstable to convection at the centre.

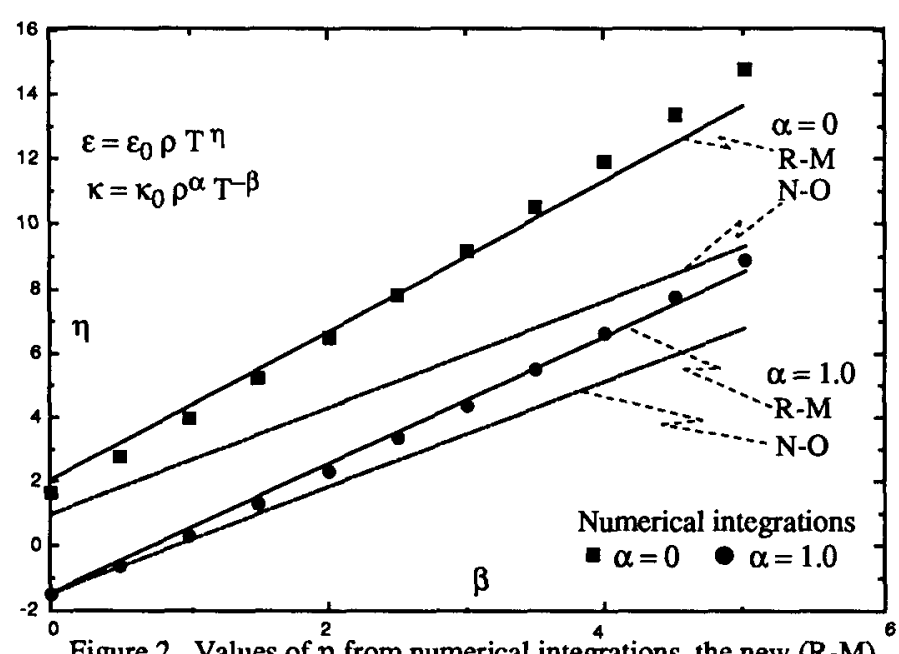

Figure 2. Values of $\eta$ from numerical integrations, the new (R-M) condition and old Naur Osterbrock ( $\mathrm{N}-\mathrm{O})$ condition.

\section{References}

Naur, P., and Osterbrock, D.E.: 1953, "Convective Cores in Stars", Astrophys J. 117, 306.

Roxburgh, I,W,: 1985, "Present Problems of the Solar Interior", Solar Physics, 100, 21. 